\title{
WHY THE LUMBRICAL MUSCLE SHOULD NOT BE BIGGER-A FORCE MODEL OF THE LUMBRICAL IN THE UNLOADED HUMAN FINGER
}

\author{
J. N. A. L. Leijnse \\ Department of Plastic and Reconstructive Surgery, Erasmus University Rotterdam, The Netherlands
}

\begin{abstract}
The present paper investigates the forces and the stresses in the lumbrical and the other finger motors in an unloaded human finger model, with and without the ab-adduction degree of freedom of the MCP joint. Unique solutions are obtained by minimization of the maximal muscle stress calculated with a normal and a variable lumbrical physiological cross-sectional area. It is concluded that in the model with biaxial MCP joint, a stronger than normal lumbrical is not useful in unloaded finger control, and will merely result in spare lumbrical capacity. Also the natural synergism of the lumbrical and the ulnar interosseus in the control of the finger in the sagittal plane is pointed out. (C) 1997 Elsevier Science Ltd. All rights reserved
\end{abstract}

Keywords: Finger model; Lumbrical muscle; Hand biomechanics.

\section{NOMENCLATURE}

\begin{tabular}{|c|c|}
\hline MCP & metacarpophalangeal joint \\
\hline PIP & proximal interphalangeal joint \\
\hline DIP & distal interphalangeal joint \\
\hline$P$ & (force in) flexor digitorum profundus \\
\hline$S$ & (force in) flexor digitorum superficialis \\
\hline$E$ & (force in) extensor digitorum \\
\hline$I$ & (force in) interosseus, 3DOF model \\
\hline$L$ & (force in) lumbrical \\
\hline$R$ & (force in) radial interosseus, 4DOF model \\
\hline$U$ & (force in) ulnar interosseus, 4 DOF model \\
\hline$M$ & (force in) medial slip of the extensor assembly \\
\hline$T$ & (force in) terminal slip of the extensor assembly \\
\hline$P_{\mathrm{d}}$ & $\begin{array}{l}\text { (force in) deep flexor tendon distal to the origin of the } \\
\text { lumbrical }\end{array}$ \\
\hline$X_{\mathrm{L}}$ & $\begin{array}{l}\text { ratio of the lumbrical relative to the deep flexor force } \\
(=L / P)\end{array}$ \\
\hline $\pm r_{i j}$ & $\begin{array}{l}\text { anatomic moment arm, positive for extension, of motor } \\
i \text { at joint } j ; 1,2,3=\mathrm{MCP}, \text { PIP, DIP }\end{array}$ \\
\hline $\pm r_{i j}^{*}$ & $\begin{array}{l}\text { systemic moment arm of motor } i \text { at joint } j \text {, positive for } \\
\text { extension }\end{array}$ \\
\hline $\begin{array}{l}r_{A R}, r_{A U} \cdot r_{A L} \\
\mathbf{R}_{i}\end{array}$ & $\begin{array}{l}\text { abduction moment arms at MCP of } R, U, L \\
\text { moment arm vector of the motor } i \text {, defined by the mo- } \\
\text { ment arms }\left(r_{i 1}, r_{i 2}\right)\end{array}$ \\
\hline$c_{i k}^{(*)}$ & $= \pm r_{11}^{(*)} \cdot\left( \pm r_{k 2}^{(*)}\right)-\left[ \pm r_{2}^{(*)} \cdot\left( \pm r_{k 1}^{(*)}\right)\right]$ \\
\hline $\mathbf{P C A}_{1}$ & normal physiological lumbrical cross-section (Table 2 \\
\hline $\mathrm{PCA}_{\mathrm{L}}$ & lumbrical cross-section as an optimization variable \\
\hline $\mathrm{PCA}_{\mathrm{LO}}$ & minimax lumbrical cross-section with $S=0$ \\
\hline
\end{tabular}

Greek letters

$\begin{array}{ll}\theta_{j} & \text { angle of joint } j, \text { positive for flexion } \\ \sigma_{\mathrm{m}} & \text { minimax stress with } \mathrm{PCA}_{\mathrm{Lp}} \\ \sigma_{0} & \text { minimax stress with } \mathrm{PCA}_{\mathrm{L} 0} \text { at } S=0 \\ \sigma_{\mathrm{p}} & \text { physiologically optimal muscle stress }\end{array}$

\section{INTRODUCTION}

The lumbrical is a relatively small finger motor with a physiological cross-sectional area of about a tenth of

\footnotetext{
Received in final form 5 August 1997.

Address correspondence to: J. N. A. L. Leijnse, Room Ee 1642, BNT, Faculty of Medicine, Erasmus University Rotterdama, P.O. Box 1738,
} 3000 DR Rotterdam, The Netherlands. that of the interossei (Brand, 1985; Chao et al., 1989). The question presently investigated is whether such a weak finger motor can play an effective role in finger control, or, conversely, whether a stronger lumbrical would be useful in the finger. To this end, the muscle stresses in static unloaded equilibrium are calculated in a finger model with four degrees of freedom: DIP(1), PIP(1), MCP(2: flexion/extension, ab-adduction), and optimized for minimal maximal muscle stress. To the biarticular lumbrical models (monoaxial MCP and PIP joints) of Thomas et al. (1968) and Ghista et al. (1978) the present study adds the abduction degree of freedom of the MCP, the third finger joint (DIP), the effect of PIP flexion on the muscle forces, and the calculation of the motor stresses. The present paper is a direct sequel to the paper of Leijnse and Kalker (1995), which investigated the kinematics of the lumbrical muscle in a similar finger model with monoaxial MCP joint.

\section{MATERIALS AND METHODS}

\section{The model}

The four degrees-of-freedom model (4DOFM) (Fig. 1) consists of the torque equilibrium equations of a biaxial MCP joint (flexion-extension, ab-adduction), and PIP, DIP hinge joints (for symbols, see Nomenclature):

$$
\begin{array}{r}
r_{E 1} \cdot E-r_{U 1} \cdot U-r_{R 1} \cdot R-r_{L 1} \cdot L-r_{S 1} \cdot S-r_{P 1} \cdot P_{\mathrm{d}}=0 \\
r_{M 2} \cdot M+r_{T 2} \cdot T-r_{S 2} \cdot S-r_{P 2} \cdot P_{\mathrm{d}}=0 \\
r_{T 3} \cdot T-r_{P 3} \cdot P_{\mathrm{d}}=0 \\
r_{A R} \cdot R-r_{A U} \cdot U+r_{A L} \cdot L=0 \\
T+M-E-U-R-L=0 \\
(1 \mathrm{~b}) \\
P-P_{\mathrm{d}}-L=0 \\
E, L, U, R, T, M, S, P, P_{\mathrm{d}} \geqslant 0
\end{array}
$$




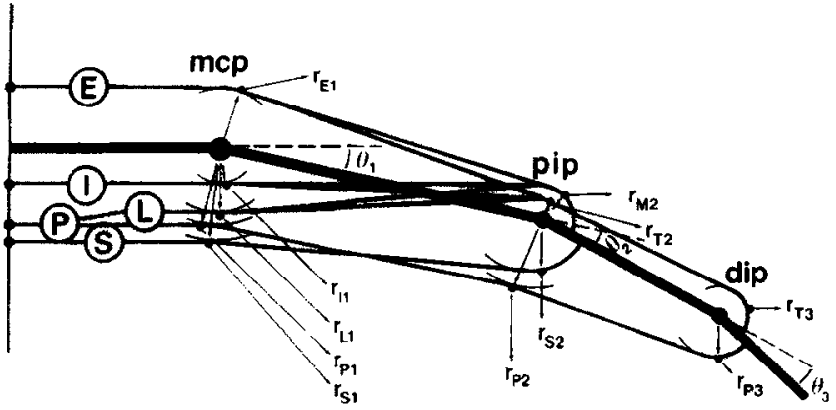

Fig. 1. Representation of the finger model in the sagittal plane.

Table 1. Moment arms (mm) of the tendons used to calculate the results

\begin{tabular}{lcccccc}
\hline MCP & $r_{P 1}$ & $r_{S 1}$ & $r_{E 1}$ & $r_{U 1}$ & $r_{R 1}$ & $r_{L 1}$ \\
& 11 & 13 & 9 & 6 & 6 & 9 \\
$\operatorname{PIP}$ & $r_{P 2}$ & $r_{S 2}$ & $r_{M 2}$ & $r_{T 2}$ & & \\
& 10.5 & 9 & 5 & $5 / 0$ & & \\
$\operatorname{DIP}$ & $r_{P 3}$ & $r_{T 3}$ & & & & \\
& 6 & 4 & & & &
\end{tabular}

Note. From: Spoor (1983). $r_{T 2}: 5$ with extended PIP; 0 with flexed PIP.

and is valid when the passive joint structures (volar plates, etc.) are slack. Moment arms of flexion and of ulnar abduction are with negative sign. The abduction moment arms of extensor and flexors at the MCP are zero. Equations(1a)-(1d) are the torque equilibrium equations of MCP, PIP and DIP in the sagittal and abduction plane; equation (1e) expresses the continuity of $E, U, R, L$ with the extensor assembly (Spoor, 1983); equation (1f) expresses the continuity of the deep flexor $P$ with $P_{\mathrm{d}}$ and $L$; conditions (1g) state that muscles can only pull, not push. Except for $r_{T 2}$ and $r_{U 1}$, all moment arms are taken constant (values in Table 1). $r_{T 2}$ is a function of the PIP position (Garcia-Elias et al., 1991), in the calculations the approximation of Leijnse and Kalker (1995) is used; with extended PIP $r_{T 2}=r_{M 2}$, and with $90^{\circ}$ flexed PIP $r_{T 2}=0$. The MCP flexion moment arms of the interossei change with the MCP position (Chao et al., 1989). The model is calculated for extended MCP with $r_{U 1}=0$ and $r_{U 1}=6 \mathrm{~mm}$; for flexed MCP with $r_{U 1}=6 \mathrm{~mm}$. Variations of $r_{R 1}$ with the MCP position, or partial insertions of the radial interosseus in the proximal phalanx are not considered. For comparison, the forces and stresses in the three degrees-of-freedom model (3DOFM) (finger in the sagittal plane) are also calculated. The 3DFOM is obtained by putting $r_{U_{1}}=r_{R 1}(=6 \mathrm{~mm})$ in equation (1a); $I=R+U$ in equations (1a) and (1e) and $r_{A V}=$ $r_{A R}=r_{A L}=0$ in equation (1d).

\section{Optimization criterion}

In the 4DOFM six motors are present $(F, P, S, R, U$, $L$ ), while four motors suffice for basic control of the unloaded finger with biaxial MCP joint. Redundancies exist with the flexors $S$ and $P$, and with $R$ and $L$. To obtain a unique solution the maximal muscle stress $\sigma_{\max }$
Table 2. Physiological cross-sectional ared of the finger motors, in $\mathrm{cm}^{*}$ (from Chao et al., 1989)

\begin{tabular}{lcccccc}
\hline Muscle & $P$ & $S$ & $E$ & $U$ & $R$ & 1. \\
\hline PCA $_{\mathrm{ip}}$ & 4.1 & 4.2 & 1.7 & 2.8 & 2.2 & 0.2 \\
\hline
\end{tabular}

is minimized, as described by An et al. (1984):

$$
\operatorname{Min} \sigma_{\max } \text { with } \sigma_{i}=M_{i} / \mathrm{PCA}_{i} \leqslant \sigma_{\max }
$$

with $M_{i}$ the muscle forces subjected to conditions (1), and $\mathrm{PCA}_{i}$ the physiological cross-sectional area of the motors $M_{i}$ (Chao et al., 1989; Table 2). The model is optimized with fixed and variable lumbrical physiological crosssectional area $\mathrm{PCA}_{\mathbf{L}}$, all other cross-sections are taken constant. The minimax stress criterion is chosen because it allows to relate the lumbrical stress, which is the object of this study, directly to the maximal stresses as normally occur in the other motors in static equilibrium.

\section{MODEL RESULTS AND PARAMETER ANALYSIS}

\section{Muscle forces in the 3DOFM and 4DOFM}

Figures 3(a) and (b) give the forces in the 3DOFM, and Fig. 4(a) and (b) in the 4DOFM, for the extended and flexed PIP, with $r_{U 1}=6 \mathrm{~mm}$. Only the 4DOFM results are commented, except when comparison is useful. Figure 4(c) gives the forces with $r_{U 1}=0 \mathrm{~mm}$.

The lumbrical force $L$ is maximal when $R=0$. The lumbrical moment arm $r_{A L}$ of MCP abduction is nonzero. Therefore, $U>0$ when $L>0$. When $R=0, U$ is only determined by the MCP abduction equilibrium: $U=r_{A L} / r_{A V} L \approx 5 / 6 L(83 \%)$. However, the contribution of the ulnar interosseus to finger equilibrium in the sagittal plane is less, because it is mechanically less effective than the lumbrical. This is illustrated by Fig. 2. Line 1 is $L / P$ in the 3 DOFM with $I=0$ and $S=0$. Line 3 is $L / P$ in the 4DOFM with $S=0, R=0$, and $r_{U 1}=r_{R 1}=$ $6 \mathrm{~mm}$. With $I=U+R$, then $E, L$ and $P$ are equal in

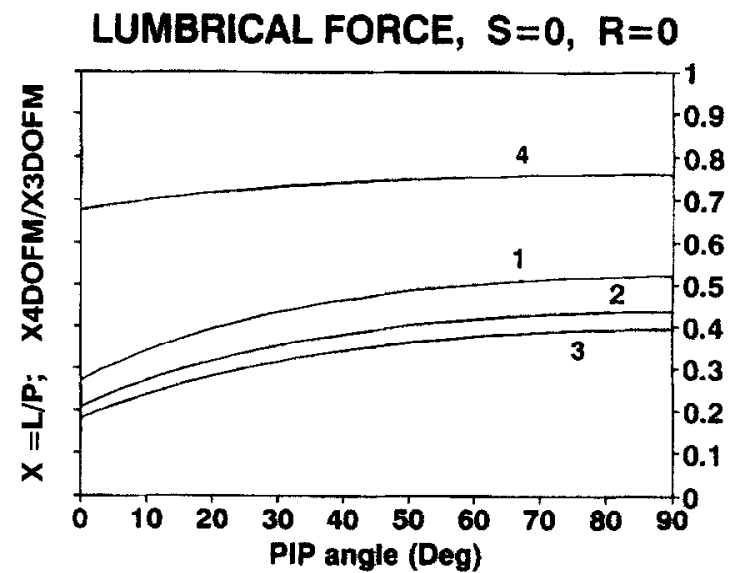

Fig. 2. Maximal lumbrical force relative to the deep flexor force as a function of the PIP position. Line 1: 3DOFM, $r_{I 1}=6 \mathrm{~mm}$. Line 2: 4DOFM, $r_{U_{1}}=0 \mathrm{~mm}$. Line 3: $r_{U_{1}}=6 \mathrm{~mm}$. Line 4: $X_{4 \mathrm{DOFM}} / X_{3 \mathrm{DOFM}}$ $(=$ line 3 /line 1): maximal relative contribution of the lumbrical to the total intrinsic force in the 4DOFM with $r_{U_{1}}=6 \mathrm{~mm}$. 
both models. The ratio of line 3 over line 1 then gives the ratio of the maximal lumbrical contribution (at $R=0$ ) to the total intrinsic function required in the $4 D O F M$ as a function of the PIP position: $68-76 \%$, (Fig. 2, line 4). The remainder $(32-24 \%)$ is provided by the ulnar interosseus. When $r_{U 1}$ decreases, the effectiveness of the ulnar interosseus in sagittal equilibrium decreases, so that the lumbrical force at $R=0$ is greater (for $r_{U 1}=0$, $L_{\max } / P$ is given by line 2 in Fig. 2).

Minimax muscle stress in the unloaded 4DOFM model with normal and variable lumbrical cross-section

The optimization process is graphically visualized in Figs 3(b), (d) and 4(b), (d) and (f), which present the muscle stresses as a function of the lumbrical force (with $P=1 \mathrm{~N}$ ), for extended and flexed PIP joint. With no condition on the force $S, S$ is minimized to zero. At $X_{L}=0$, the extensor, which alone balances all other motor forces at the MCP and which has a relatively small cross-section, determines the maximal stress. With increasing lumbrical force $\left(X_{L}>0\right)$, the extensor stress $\sigma_{E}$ decreases, but the lumbrical stress $\sigma_{L}$ increases much faster because of the small $\mathrm{PCA}_{\mathrm{Lp}}$. The minimax stress is reached when either $\sigma_{L}=\sigma_{E}$, or when the positivity conditions $(1 \mathrm{~g})$ are violated. With the normal lumbrical $\mathrm{PCA}_{\mathrm{Lp}}$, in all models and positions with $S$ minimized to zero, $\sigma_{L}=\sigma_{E}$ is reached well before violation of condition $(1 \mathrm{~g})$, producing the physiological minimax stress $\sigma_{\mathrm{m}}$, at $X_{\mathrm{m}}$. However, $\sigma_{E}$ can be further reduced while keeping the minimax stress condition $\sigma_{E}=\sigma_{L}$ satisfied by increasing the lumbrical force $L$ while decreasing $\sigma_{L}=L / \mathrm{PCA}_{\mathrm{L}}$ by increasing the lumbrical cross-section $\mathrm{PCA}_{\mathrm{L}}$. This corresponds to rotating the lumbrical stress line $L / \mathrm{PCA}_{\mathrm{L}}$ clockwise about zero in the stress diagrams. The resulting overall minimax stress $\sigma_{0}$ is reached when $R=0$ (at $X_{0}$ ), with a lumbrical crosssection $\mathrm{PCA}_{\mathrm{L} 0}=L / \sigma_{0}$. Any further increase in $\mathrm{PCA}_{\mathrm{L}}$ will then lead to a lumbrical stress smaller than the extensor stress. For all cases $\sigma_{\mathrm{m}}>\sigma_{0}(S=0)$, so it holds that $\mathbf{P C A}_{\mathbf{L p}}<\mathrm{PCA}_{\mathbf{L O}}$. Table 3 shows that this difference is less in the extended than in the flexed finger. In the extended model with $r_{U 1}=6 \mathrm{~mm}$ the difference is smallest $\left(\mathrm{PCA}_{\mathrm{L} 0}=1.3 \mathrm{PCA}_{\mathrm{Lp}}\right)$. When the lumbrical force $X_{L}$ exceeds $X_{0}$, it must be that $S>0$, otherwise $R<0$ (violation of equation $(1 \mathrm{~g})$ ). $E$ increases with $S$ at a far greater rate than the interossei or lumbrical forces.
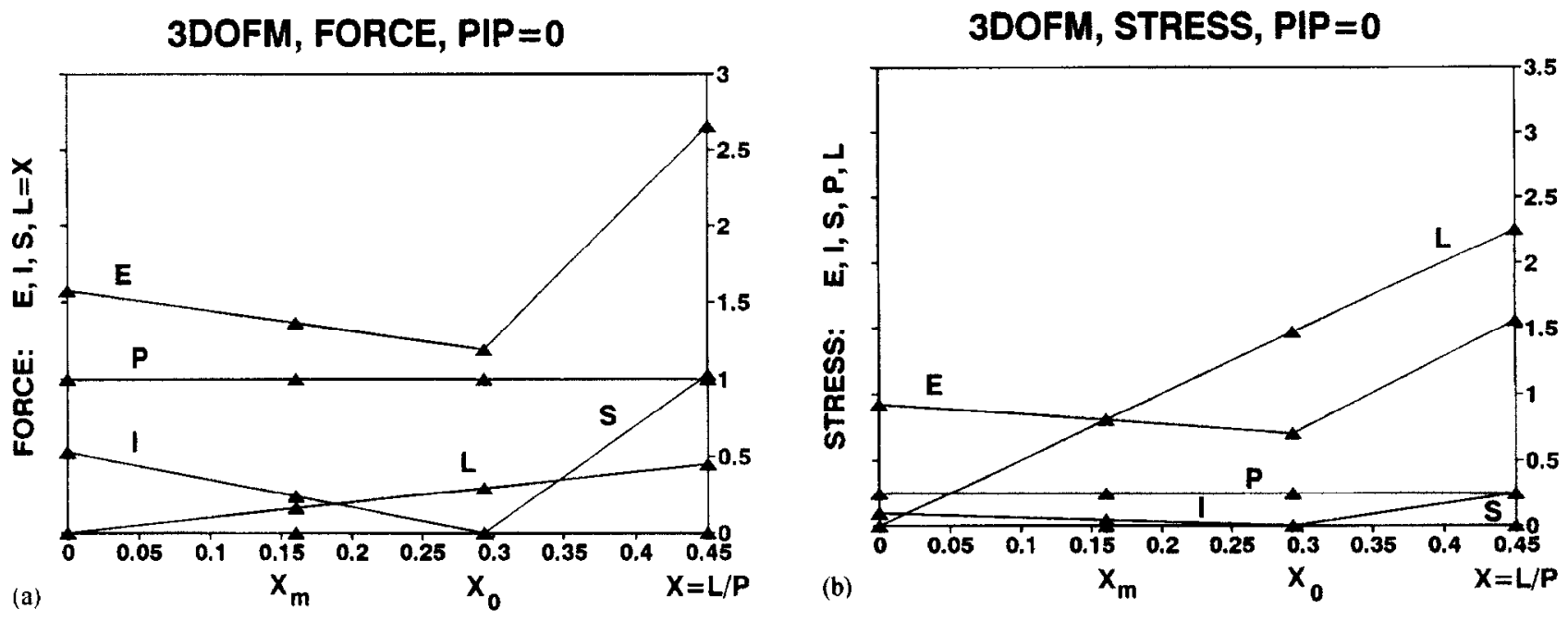

3DOFM, FORCE, PIP $=90$

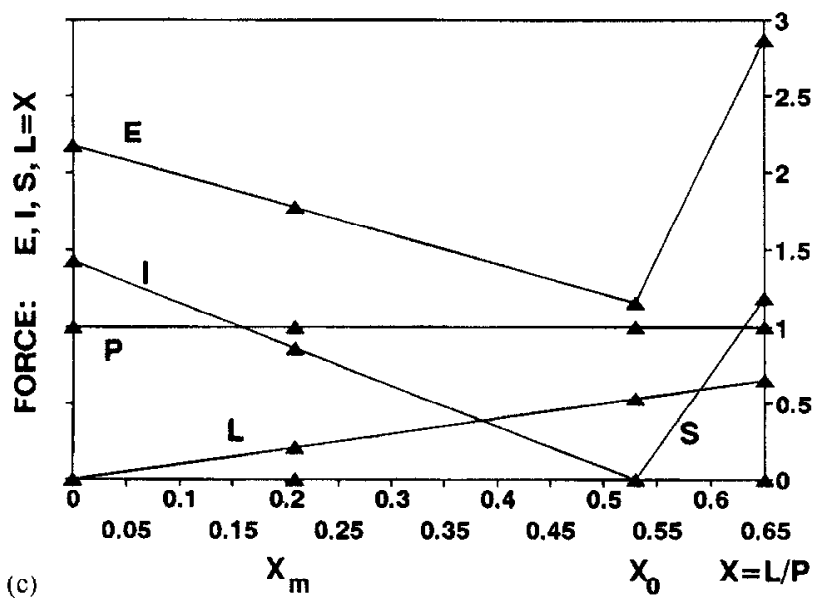

\section{DOFM, STRESS, PIP $=90$}

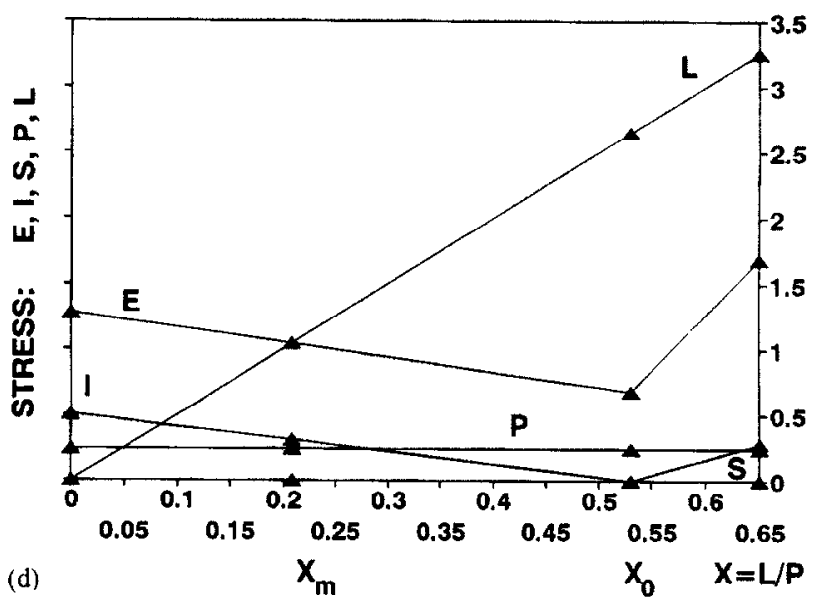

Fig. 3. Forces and stresses $\left(\mathrm{N} \mathrm{cm}^{-2}\right)$ in the $3 D O F M$ as a function of the relative lumbrical force $X=L / P$.

(a) and (b). Forces, stresses, extended finger. (c) and (d). Forces, stresses, flexed finger. 

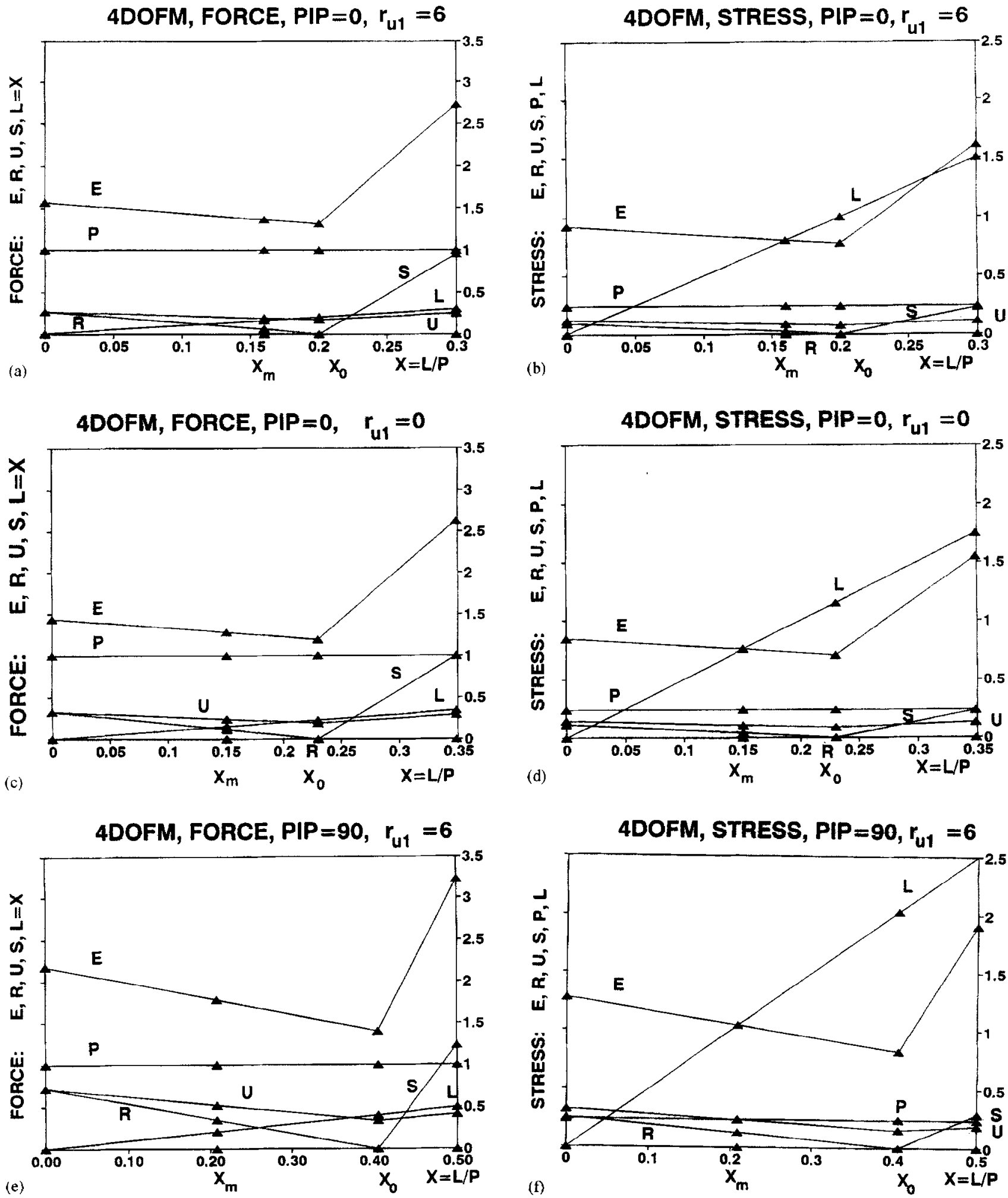

Fig. 4. Forces and stresses $\left(\mathrm{N} \mathrm{cm}^{-2}\right)$ in the 4 DOFM as a function of the lumbrical force $(X=L / P)$ (a) and (b) Forces, stresses, extended finger, $r_{U 1}=6 \mathrm{~mm}$; (c) and (d) Forces, stresses, extended finger, $r_{U 1}=0 \mathrm{~mm}$; (e) and (f) Forces, stresses, flexed PIP, $r_{U 1}=6 \mathrm{~mm}$.

With $S>0, P>0$, and $R=0$, in the flexed 4DOFM with normal lumbrical $P_{C A} A_{L p}$ the lumbrical stress far exceeds the second greatest stress $\sigma_{E}\lceil$ Fig. 4(f) $\rceil$. Therefore, in the flexed 4DOFM with $S \geqslant 0$ the minimax stress $\sigma_{L}=\sigma_{E}$ will be with $R>0$. With extended PIP (Fig. 4(b) and (d)), the physiological lumbrical stress and the extensor stress at $R=0$ are closer, especially for greater $S$. Even, with $r_{U 1}=6 \mathrm{~mm}$ and $R=0$, for $S>0.6 P$ the physiological lumbrical stress is less than the extensor stress [Fig. 4(b)]. With extended PIP, $r_{U 1}=0$, and 
Table 3. Ratio of the optimal and the normal lumbrical cross-sections $\left(\mathrm{PCA}_{\mathrm{LD}} / \mathrm{PCA}_{\mathrm{Lp}}\right)$ in the 3DOFM and 4DOFM, for the extended $(e)$ and flexed $(f)$ finger, with $S=0$ and $S=P$, and different values of $r_{U 1}$ (in Inm)

\begin{tabular}{lccccc}
\hline & $\begin{array}{l}3 \mathrm{DOF}_{e} \\
r_{11}=6\end{array}$ & $\begin{array}{l}3 \mathrm{DOF}_{f} \\
r_{f 1}=6\end{array}$ & $\begin{array}{l}4 \mathrm{DOF}_{e} \\
r_{U 1}=6\end{array}$ & $\begin{array}{l}4 \mathrm{DOF}_{e} \\
r_{U 1}=0\end{array}$ & $\begin{array}{l}4 \mathrm{DOF}_{f} \\
r_{U 1}=6\end{array}$ \\
\hline$S=0$ & 2.1 & 3.7 & 1.3 & 1.64 & 2.5 \\
$S=P$ & 1.5 & 2 & 0.9 & 1.15 & 1.4 \\
\hline
\end{tabular}

$S=P$, then $\sigma_{\mathrm{Lp}} / \sigma_{\mathrm{E}}=1.15 \quad$ [Fig. 4(d)]. The ratios $\sigma_{\mathrm{Lp}} / \sigma_{\mathrm{E}}$ with $S=P$ and $R=0$ are summarized in Table 3 (it holds that, with fixed $S>0$ and $R=0, \sigma_{\mathrm{Lp}} / \sigma_{\mathrm{E}}=$ $\mathrm{PCA}_{\mathrm{L} \delta} / \mathrm{PCA} A_{\mathrm{Lp}}$ ).

\section{DISCUSSION}

A physical interpretation of the minimax stress criterion - the lumbrical-extensor synergism

To allow a physical interpretation of. the minimax stress criterion, the concept of 'spare motor capacity' is introduced. Minimization of muscle stress with variable cross-sections results in finite cross-sections only when a penalty on spare motor capacity is imposed. Spare motor capacity in a given task can be defined as a muscle stress smaller than a physiologically optimal muscle stress $\sigma_{\mathrm{po}}$. With a penalty on such spare capacity, minimizing stress in muscles $M_{i}$ with variable cross-sections for a single task (e.g. unloaded static finger equilibrium) results in $\sigma_{i}=\sigma_{\mathrm{po}}$ for all muscles, since cross-sections initially being too small $\left(\sigma_{i}>\sigma_{\mathrm{po}}\right)$ increase to minimize the muscle stress, while those too large $\left(\sigma_{i}<\sigma_{\mathrm{po}}\right)$ decrease to minimize the penalty. However, minimizing muscle stress for multiple tasks will result in some motors, possibly all, having spare capacity $\left(\sigma_{i}<\sigma_{\text {po }}\right)$ in individual tasks, while for each motor tasks will exist in which $\sigma_{i}=\sigma_{\mathrm{po}}$. The latter tasks then determine the crosssections of these motors. Within this view, the following interpretation of the results can be envisaged.

(i) In the unloaded finger, the minimax stress is set by the extensor, which is the only extensor of the MCP and cannot escape load. For given $S$ and $P$, the extensor stress is minimized by increasing $L$ within the ranges of equation $(1 \mathrm{~g})$.

(ii) Relative to the extensor stress $\sigma_{E}$, the motors, $P, S$, $U, R$ have considerable spare capacity $\left(\sigma_{\mathrm{p}}, \sigma_{\mathrm{s}}, \sigma_{U}\right.$, $\sigma_{R} \ll \sigma_{E}$ ), meaning that their cross-sections are determined by loaded instead of unloaded tasks.

(iii) With PIP flexion, the forces $E, U, R$ and $L$ increase relative to the flexor forces, due to the decrease of the moment arm of the terminal slips at the PIP with PIP flexion. However, this relative force increase does not mean that in reality the stress in $E, R, U$ and $L$ becomes greater in absolute terms. In the extended finger, the passive flexor forces in the stretched flexors are large. With flexed PIP, the passive flexor forces are small, and the active flexor forces required for unloaded finger control need not be large. This suggests that the mechanical effectiveness of the extensor apparatus is optimized for the extended finger, where the greatest absolute forces occur.

(iv) Consider the extended finger, in which $S>0$, because of its passive stretching. With $S>0$, and $R=0$, the physiological lumbrical stress is only a little greater, or even smaller [Fig. 4(b)] then the extensor stress. This suggests that the lumbrical $\mathrm{PCA}_{\mathrm{I}}$ is 'tuned' to the extensor $\mathrm{PCA}_{E}$ in the task of unloaded finger extension, implying that the lumbrical and the extensor are natural synergists in this task. In addition, the fact that the other motor stresses are much smaller, that the mechanical effectiveness of the extensor assembly is optimal with extension, and that finger extension is an important hand function suggests that the extensor $\mathbf{P C A}_{E}$ is itself determined by the task of unloaded finger extension. If this holds, the equality between the extensor and lumbrical stress in the extended finger should not be considered as accidental, but the result of an optimization of their PCA for this task.

\section{The effect of the lumbrical force on interosseus} and extensor force and stress

The interossei have considerable spare capacity relative to the extensor, even with $L=0$ [Fig. 4(b), (d) and (f)]. Moreover, the decrease in ulnar interosseus force with lumbrical force is small, especially in the extended finger [Fig. 4(b) and (d)]. In the extended finger, the extensor stress reduction with $L$ is also small $[<10 \%$, Fig. 4(b) and (d)], while with flexed PIP it is potentially greater but requires a physiological lumbrical stress much in excess of the extensor stress [Fig. 4(f)]. Therefore, the prime function of the lumbrical in the unloaded finger can hardly be the static unloading of the interossei or extensor, which suggests that a further role may be found in control of unloaded finger dynamics. The mechanism of unloading of the extensor is twofold [equation (1a)]: (i) the greatest effect is the reduction of the interosseus flexion moment at the MCP by lumbrical substitution of $R$ and $U$; and (ii) a smaller, almost insignificant effect is that the lumbrical reroutes force from the deep flexor $P$ at the MCP with a slightly smaller flexion moment arm.

The effect of the lumbrical force on the relative forces in the medial and terminal slips

The lumbrical partly unloads the terminal slips of the extensor assembly, as the force $T$ in the terminal slips decreases in proportion to the reduction of $P_{\mathrm{d}}$ relative to the total deep flexor force $P$ [equation (1c)]. For example, in the extended models with $S=P, L$ reduces $T$ by about a third [Fig. 4(b) and (d)]. This corresponds to the concept that the lumbrical's function is to pull the deep flexor tendons distalwards against their passive force with finger extension (Long, 1968). However, since $E$ is not reduced to the same degree, the force $M$ in the medial slip will increase with the decrease in $T$ [equation (le)]. In other words, $L$ transfers force from the terminal to the medial extensor slip.

\section{The superficial flexor force in the unloaded finger}

Minimizing muscle stress with non-zero deep flexor force results in $S=0$. This indicates that the use of $S$ in the static unloaded finger is non-optimal: $S$ increases the stress in extensor and intrinsics, but provides no function which the deep flexor cannot provide in the unloaded finger with active PIP-DIP coupling mechanism (Leijnse, 1997). In the flexed finger, where the passive 
Hexor forces are low, $P \gg S \approx 0$ may be realistic. However, in the extended finger, with high passive forces. $S$ approaching $P$ seems realistic. $S$ loads the extensor far more than the intrinsics, because the superficial flexor and extensor are almost exact antagonists (Leijnse, 1997) [Appendix A, Fig. Al(c)]. Therefore, with $S>0$ and $R=0$, the lumbrical will not incur stresses much in excess of the extensor stress, especially when $S$ approaches $P$ (with extended MCP, realistically, it holds that $0<r_{U 1}<6 \mathrm{~mm}$. With $S=P$, and $r_{U 1}=6$ and $r_{U 1}=0 \mathrm{~mm}, \sigma_{\mathrm{Lp}}=0.93 \sigma_{\mathrm{E}}$ and $\sigma_{\mathrm{Lp}}=1.15 \sigma_{\mathrm{E}}$, resp.).

\section{Should the lumbrical be bigger?}

According to the above, the greatest motor stresses occur in the extended finger, and in the extended 4DOF model the minimax lumbrical stress is about equal to the extensor stress. This suggests that a stronger-than-normal lumbrical would merely result in surplus lumbrical capacity. This is not the case in the extended $3 \mathrm{DOF}$ model [Fig. 3(b)], where $\sigma_{\mathrm{Lp}} / \sigma_{\mathrm{E}}=2 \ldots 1.5(0 \leqslant S \leqslant P)$. The difference in these results is due to the ulnar interosseus, which in the 4DOF model is the lumbrical antagonist for MCP abduction, but which is its synergist in the sagittal plane. This suggests that in a real finger the lumbrical strength is tuned to the extensor strength, taking into account the synergistic action of the ulnar interosseus. With paralysis of the interossei the finger functions as the 3DOF model, the radial abduction being checked by the ulnar collateral MCP ligaments in some MCP abduction end position. In the 3DOF model with $I=0$ the physiological lumbrical stress in unloaded equilibrium is at least $30 \%$ greater than in the 4DOF model. This excess strain, and the fact that the lumbrical without interossei will also be recruited in loaded tasks for which it is not designed, may explain why without interossei but with intact lumbricals clawing in fingers over time may still develop, as a result of lumbrical overuse.

\section{CONCLUSION}

A four degree of freedom static force model of the unloaded finger with lumbrical and with slack palmar joint ligaments was investigated, leading to the following conclusions.

(i) The mechanical effectivity of the extensor $(E)$ and intrinsics $(U, R, L)$ decreases relative to the deep flexor $(P)$ with PIP flexion. It is greatest in the extended finger, where the passive flexor forces are maximal.

(ii) In the unloaded finger, a superficial flexor is not required for equilibrium and leads to greater muscle forces than minimally required, especially in the extensor.

(iii) In the unloaded model with MCP in neutral position of abduction, the radial abduction moment of the lumbrical must be balanced by the ulnar interosseus. However, this interosseus force is synergistic with the lumbrical in finger equilibrium in the sagittal plane. The lumbrical may provide, maximally, about $70 \%$ of the total required intrinsic function, otherwise the radial interosseus force would have to be negative. The remaining intrinsic function is then provided by the ulnar interosseus. Without interossei, the finger radially deviates at the MCP until retained by passive structures (MCP ligaments). The lumbrical may then provide all intrinsic function and prevent finger clawing, but its stress will be at least $30 \%$ greater than with functional interossei.

(iv) The unloaded model with normal interossei becomes unbalanced when the lumbrical force exceeds about a third of the force of the deep flexor in the extended finger, and about half of the deep flexor force in the flexed finger. Assuming that the greatest absolute flexor forces occur in the extended finger. due to the passive stretching of the flexors, the lumbrical should not be stronger than required to pull a third of the passive deep flexor force.

(v) In the extended model, the extensor stress and the maximal lumbrical stress are about equal. This suggests that the lumbrical strength is tuned to the extensor strength for the range of near extension, where the passive flexor forces are highest. Assuming that the role of the lumbrical is indeed with unloaded finger extension, and that the extensor stress in this task is a physiologically normal one, it can be concluded that a stronger than physiological lumbrical is not useful, and would merely lead to spare lumbrical muscle capacity.

Acknowledgements-The author cordially thanks Dr C. W. Spoor, Prof, Emer. J. M. F. Landsmeer, Prof. C. J. Snijders, Prof. J. J. Van Der Meulen, Dr G. J. Sonneveld and Dr S. E. R. Hovius for their valuable contributions, and the ANNA funds for its sponsorship.

\section{REFERENCES}

An, K. N., Kwak, B. J., Chao, E. Y. and Morrey, B. F. (1984) Determination of muscle and joint forces: a new technique to solve the indeterminate problem. Journal of Biomedical Engineering 106, 364-367.

Brand, P. W. (1985) Clinical Mechanics of the Hand, pp. 194-195. C. V. Mosby, St. Louis.

Chao, Y. S. An, K. N., Cooney, III W. P. and I inscheid, R. I. (1989) Biomechanics of the Hand; a Basic Research Study, pp. 24-45. World Scientific, Singapore.

Garcia-Elias, M., An, K. N., Berglund, L., Linscheid, R. L., Cooney. W. P. and Chao, E. Y. S. (1991) Extensor mechanism of the fingers. I. A quantitative study. Journal of Hand Surgery 16A, 1130-1136.

Ghista, D. N., Kasturirangan, S., Jayaraman, G. and Srinıvasan, H. (1978) Mechanics of normal and claw hands: analysis and guidelines for surgical correction of the claw hand. In Orthopedic Mechanics - Procedures and Devices, eds D. N. Ghista and R. Roaf, pp. 99-122. Academic Press, London.

Leijnse, J. N. A. L., Bonte, J. E., Landsmeer, J. M. F., Kalker, J. J., van der Meulen, J. C. and Snijders, C. J. (1992) Biomechanics of the finger with anatomical restrictions ... the significance for the exercising hand of the musician. Journal of Biomechanics 25, 1253-1264.

Leijnse, J. N. A. L. and Kalker, J. J. (1995) A two dimensional kinematic model of the lumbrical in the human finger. Journal of Biomechanics 28, 237-249.

Leijnse, J. N. A. L. (1996) A graphic analysis of the biomechanics of the bi-articular chain - application to the proximal bi-articular chain of the human finger. Journal of Biomechanics 29, 355-366.

Leijnse, J. N. A. L. (1997) The controllability of the unloaded human finger with the superficial or decp flexor. Journal of Biomechanics 30 , $1087-1093$.

Long, C. (1968) Intrinsic-extrinsic muscle control of the fingers. Journal of Bone and Joint Surgery 50A, 973-984.

Spoor, C. W. and Landsmeer, J. M. F. (1976) Analysis of the zigzag movement of the human finger under influence of the extensor digitorum tendon and the deep flexor tendon. Journal of Biomechanics 9, 561-566.

Spoor, C. W. (1983) Balancing a force on the fingertip of a two dimensional finger model without intrinsic muscles. Journal of Biomechanics 16, 497-504.

Thomas, D. H., Long, C. and Landsmeer, J. M. F. (1968) Biomechanical considerations of lumbricalis behaviour in the human finger. Journal of Biomechanics 1, 107-115. 


\section{APPENDIX A}

The bi-articular equivalent 3DOF model and the moment arm vector diagram

The 3DOFM can be graphically presented in a vector diagram as defined in Leijnse (1996). The climination of the variables $M, T$, and $P_{\mathrm{d}}$ from the 3 DOFM leaves two equations:

$E \cdot\left[\begin{array}{l}r_{E 1} \\ r_{M 2}\end{array}\right]+I \cdot\left[\begin{array}{c}-r_{I 1} \\ r_{M 2}\end{array}\right]+L \cdot\left[\begin{array}{c}r_{L 1}^{*} \\ r_{L 2}^{*}\end{array}\right]+S \cdot\left[\begin{array}{c}-r_{S 1} \\ -r_{S 2}\end{array}\right]+P \cdot\left[\begin{array}{c}-r_{P 1} \\ -r_{P 2}^{*}\end{array}\right]$

$$
=\left[\begin{array}{l}
0 \\
0
\end{array}\right]
$$

in which the systemic moment arms $r_{P 2}^{*}, r_{L 1}^{*}$ and $r_{L 2}^{*}$ are given by (Spoor and Landsmeer, 1976; Leijnse and Kalker, 1995):

$$
r_{P 2}^{*}-r_{P 2}+\frac{r_{P 3}}{r_{T 3}}\left(r_{M 2}-r_{T 2}\right)
$$

$$
\begin{aligned}
& r_{L 1}^{*}=r_{P 1}-r_{L 1} \\
& r_{L 2}^{*}=r_{P 2}^{*}+r_{M 2}
\end{aligned}
$$

Equations (A1) describe the equilibrium of the equivalent bi-articular chain of Fig. Al(a). in which the lumbrical is present with a fixed origin (Leijnse and Kalker, 1995), with the condition that $L \leqslant P$. The column matrices in expression (Al) can be interpreted as the cartesian coordinates of the 'moment arm vectors' $\mathbf{R}_{E}, \ldots, \mathbf{R}_{P}^{*}$. These vectors allow to visualize the equations ( $\mathrm{A} 1$ ) in a two-dimensional vector diagram (Fig. A1(b) and (c)]. The equilibrium motor forces are the factors of vector multiplication required to obtain a zero vector sum in this diagram. From the vector diagrams in Fig. Al(b) and (c) the redundancy of interosseus and lumbrical, and of superficial and deep flexor are clear, since any convex combination of the moment arm vectors of $I$ and $L$, and of $P$ and $S$, resp., can produce a zero vector sum:

$$
\begin{aligned}
& K_{1} \cdot\left[\lambda_{1} \cdot \mathbf{R}_{P}^{*}+\left(1-\lambda_{1}\right) \cdot \mathbf{R}_{S}\right]+K_{2} \cdot\left[\lambda_{2} \cdot \mathbf{R}_{1}+\left(1-\lambda_{2}\right) \cdot \mathbf{R}_{L}^{*}\right] \\
& \quad+E \cdot \mathbf{R}_{E}=0
\end{aligned}
$$

with $0 \leqslant \lambda_{i} \leqslant 1$, and $K_{i}, E \geqslant 0$.
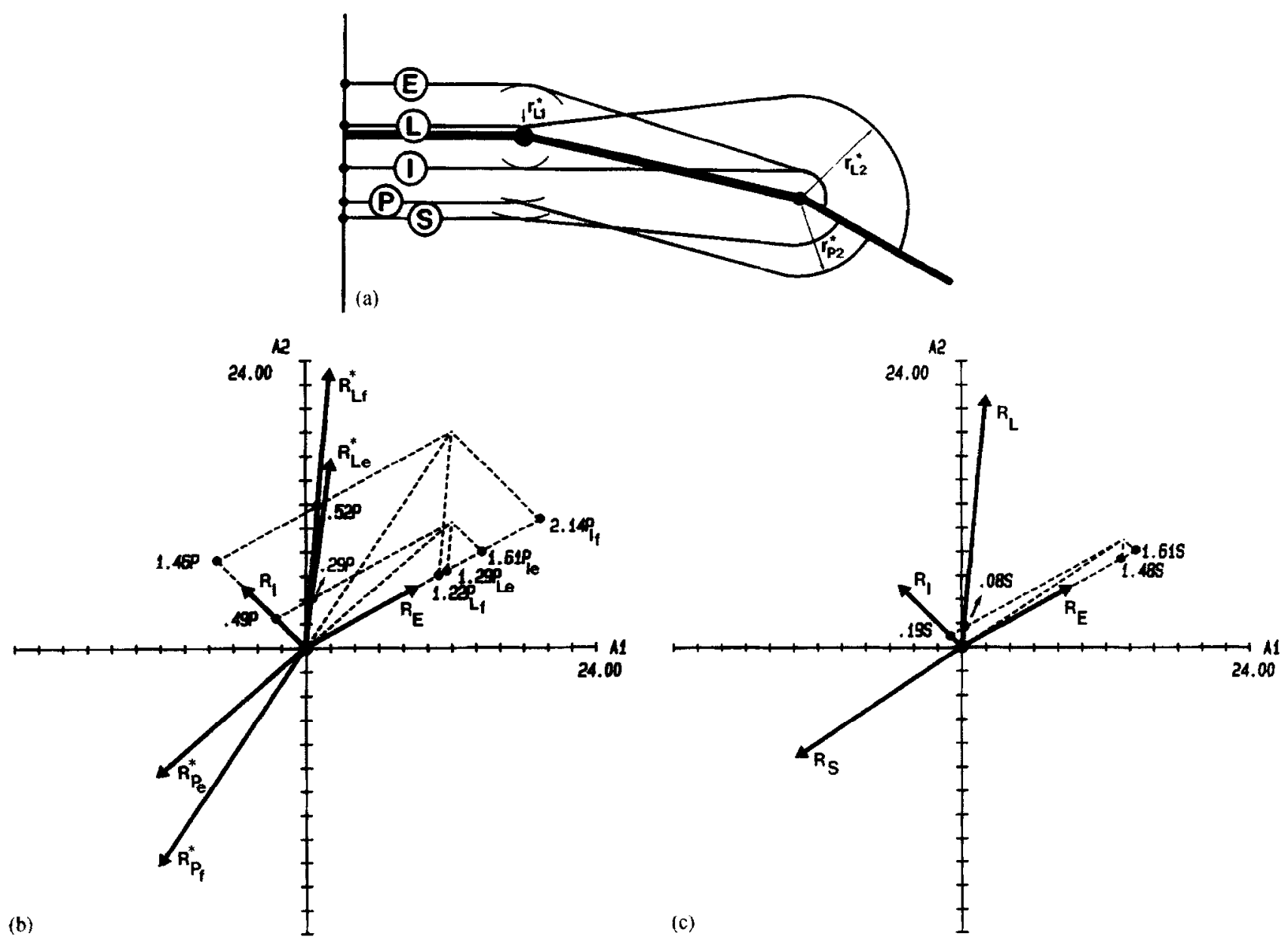

Fig. A1. (a) Bi-articular equivalent model. (b) Combined moment arm vector diagram of the two-dimensional model presenting the motor forces $E, I, L$, as a fraction of the deep flexor force $P$, with $90^{\circ}$ flexed ( $f$ ) and extended (e) PIP. Only the moment arm vectors $\mathbf{R}_{P}^{*}$ and $\mathbf{R}_{L}^{*}$ change with PIP flexion. The muscle forces are obtained as follows. With $I=0, L_{e}$ or $L_{\mathrm{f}}$ are equal to the length of the projection, parallel to $\mathbf{R}_{E}$, of the moment arm vectors ${ }^{-} \mathbf{R}_{\mathrm{Pe}}^{*}$ or ${ }^{-} \mathbf{R}_{\mathrm{Pf}}^{*}$ on $\mathbf{R}_{\mathrm{Le}}^{*}$ or $\mathbf{R}_{L \mathrm{f}}^{*}$, divided by the length of $\mathbf{R}_{\mathrm{Le}}^{*}$ or $\mathbf{R}_{\mathrm{Lf}}^{*}$, resp.; $E_{\mathrm{e}}$ and $E_{\mathrm{f}}$ with $I=0$ follow similarly from the projection, parallel to $\mathbf{R}_{L \mathrm{c}}^{*}$ or $\mathbf{R}_{L \mathrm{f}}^{*}$, of ${ }^{-} \mathbf{R}_{\mathrm{Pc}}^{*}$ or ${ }^{-} \mathbf{R}_{\mathrm{Pf}}^{*}$, resp., on $\mathbf{R}_{E}$. This results in: $L / P_{\mathrm{e}}-0.29, E / P_{\mathrm{e}}-1.29 ; L / P_{\mathrm{f}}-0.52, E / P_{\mathrm{f}}-1.22$. With $L-0, I$ is the projection, parallel to $\mathbf{R}_{E}$, of ${ }^{-} \mathbf{R}_{P_{\mathrm{e}}}^{*}$ or ${ }^{-} \mathbf{R}_{P_{\mathrm{r}}}^{*}$ on $\mathbf{R}_{I}$, divided by the length of $\mathbf{R}_{I}$; while $E$ with $L=0$ is the projection, parallel to $\mathbf{R}_{I}$, of ${ }^{-} \mathbf{R}_{P_{c}}^{*}$ or ${ }^{-} \mathbf{R}_{P \mathrm{f}}^{*}$ on $\mathbf{R}_{E}$. This results in: $I / P_{\mathrm{e}}=0.49, E / P_{\mathrm{e}}=1.61: I / P_{\mathrm{f}}=1.46, E / P_{\mathrm{f}}=2.14$. The mutual redundancy of $L$ and $I$ follows from the fact that $\mathbf{R}_{P}^{*}$ can be balanced by $\mathbf{R}_{E}$, and any appropriately scaled positive linear combination of $\mathbf{R}_{I}$ and $\mathbf{R}_{L}$. (c) The forces $E, I, L$ as a function of the superficial flexor force $S$. With $I=0$, $L / S=0.08, E / S=1.48$; with $L=0, I / S=0.19, E / S=1.61$. 


\section{APPENDIX B}

Phisical inferpetations of lumbrical fanction

Different physical interpretations of lumbrical function can be derived from the analytical solutions of the 3DOFM of expression (A1)

$$
\begin{aligned}
& I=\frac{c_{P E}^{*}}{c_{E I}} \cdot\left(1-X_{L}\right) \cdot P-\frac{c_{E L}}{c_{E I}} \cdot X_{L} \cdot P+\frac{c_{S E}}{c_{E I}} \cdot S \\
& E=\frac{c_{P}^{*}}{c_{E I}} \cdot\left(1-X_{L}\right) \cdot P+\frac{c_{E L}}{c_{E I}} \cdot X_{L} \cdot P+\frac{c_{I S}}{c_{E I}} \cdot S
\end{aligned}
$$

(in which $c_{i k}^{(*)}= \pm r_{i 1}^{(*)} \cdot\left( \pm r_{k 2}^{(*)}\right)-\left[r_{i 2}^{(*)} \cdot\left( \pm r_{k 1}^{(*)}\right)\right]$, and $\pm r_{i j}^{(*)}$ are the anatomic or systemic moment arms with sign as in the model (A1). In the following it is assumed that $S=0$.

(i) The intrinsic-extrinsic balance. With zero lumbrical force $X_{L}=0$ expression $(B 1 \mathrm{a})$ can be written as

$$
c_{E I} \cdot I=c_{P E}^{*} \cdot P \text {. }
$$

This equation gives the interosseus and deep flexor forces relative to a given extensor force, and reflects the Landsmeer view of finger control as a balance of extrinsic (clawing inducing) and intrinsic (counterclawing) actions (Leijnse and Kalker, 1995; Spoor and Landsmeer, 1976). The right-hand side represents the 'clawing-inducing' action of the extrinsics, as a product of the extrinsic force $P$ and the extrinsic 'clawing-inducing effectivity' $c_{P E}^{*}$, a factor which contains only extrinsic moment arms. The left-hand side represents the intrinsic "counterclawing' action required for equilibrium, as a product of the interosseus force and its intrinsic counterclawing effectivity $\mathbf{c}_{E I}$

(ii) Lumbrical-interosseus substitution $\left(X_{L}>0\right)$. The substitution of the interosseus by the lumbrical force in the expression (B1a) is determined by two distinct terms, each of which has a physical meaning.

(a) $c_{P E}^{*} / c_{E I}^{*} \cdot\left(1-X_{L}\right) \cdot P$ : the lumbrical force $X_{L}$ reduces the total extrinsic "collapse-inducing' force $c_{P E}^{*} \cdot P$ by the factor $\left(1-X_{L}\right)$, which may be called the 'extrinsic' lumbrical effect. This effect can mathematically be interpreted in two ways:

$$
\begin{aligned}
& {\left[c_{P E}^{*} \cdot\left(1-X_{L}\right)\right] \cdot P=c_{P E X}^{*} \cdot P,} \\
& c_{P E}^{*} \cdot\left[\left(1-X_{L}\right) \cdot P\right]=c_{P E}^{*} \cdot P_{\mathrm{d}},
\end{aligned}
$$

The first interpretation ( $\mathrm{B} 3 \mathrm{a}$ ) is in the terms of the intrinsic-extrinsic balance, and states that the lumbrical reduces the effectivity $c_{P E X}^{*}$ of the collapse-inducing action of the extrinsics. The second interpretation (B3b) corresponds to what has been named the "reduction of the elastic pull' of the deep flexor (Long, 1968): the lumbrical force reduces the force $P_{\mathrm{d}}=\left(1-X_{L}\right) \cdot P$ in the deep flexor tendon distal to the lumbrical origin.

(b) $c_{E L} / c_{E I} \cdot X_{L} \cdot P$. This term expresses the 'intrinsic effect' of the lumbrical. Similar to $c_{E I}$ (the counterclawing effectivity of the interosseus, expression (B2)], $c_{L E}$ can be seen as the effectivity of the lumbrical, when considered to be an interosseus, i.e. with a fixed origin from bone proximal to the MCP. The factor $c_{E L} / c_{l E}$ then expresses the relative effectivity of such a lumbrical and the real interosseus. This ratio is somewhat to the advantage of the lumbrical, as $r_{L 1}>r_{I 1}$, so that $c_{E L} / c_{I E}>1$

(iii) Equation (B1b) can be similarly interpreted. Extrinsic effect: the reduction in extensor force due to the extrinsic effect $\left(1-X_{L}\right)$ of the lumbrical is clear. Intrinsic effect: when the interosseus is replaced by an interosseus with lumbrical moment arms, the extensor force increases to
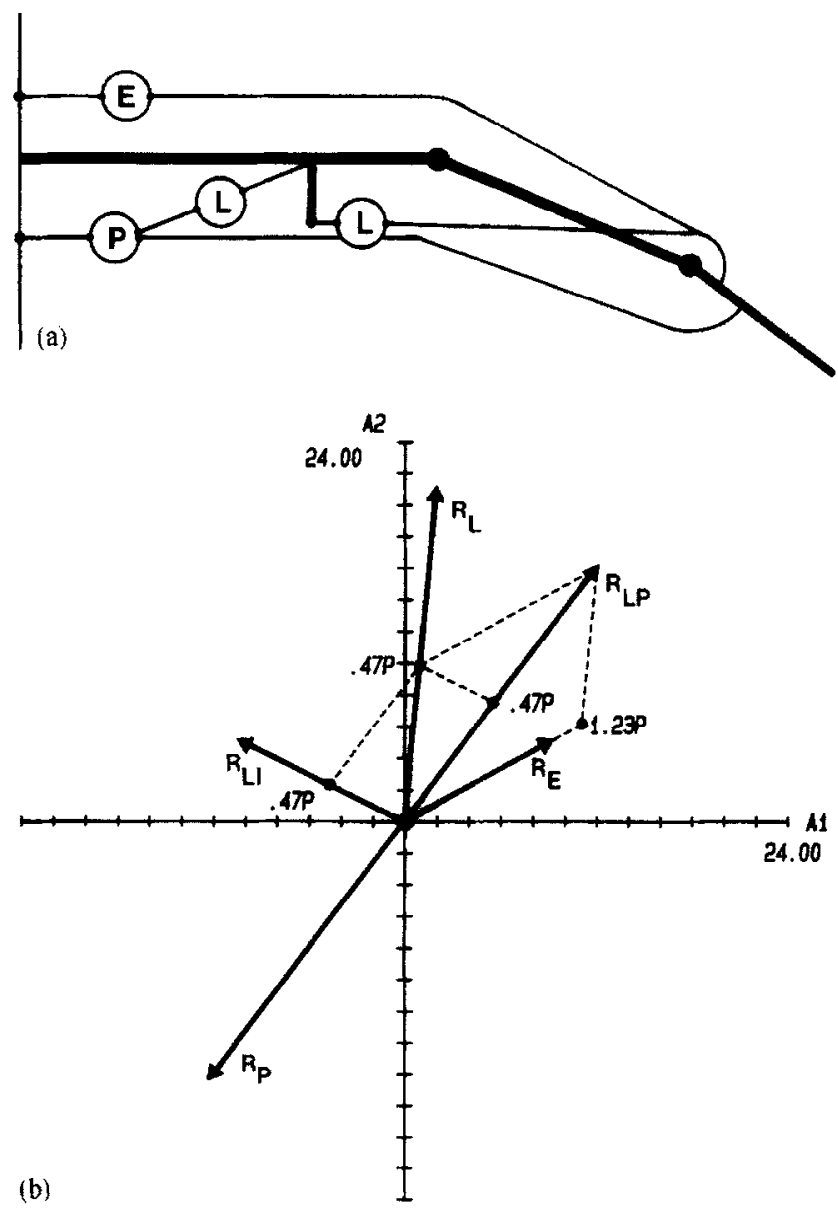

Fig. B1. (a) Lumbrical function as represented by two separate motors. (b) Vector diagram of Fig. B1(a). The lumbrical moment arm vector $\mathbf{R}_{L}^{*}$ is decomposed into the moment arm vectors of two separate motors $\mathbf{R}_{L P}^{*}$ and $\mathbf{R}_{L I} . \mathbf{R}_{L P}^{*}$ directly opposes $\mathbf{R}_{P}^{*} . \mathbf{R}_{L I}$ and $\mathbf{R}_{E}$ balance the remaining deep flexor forces. The forces in $\mathbf{R}_{L I}$ and $\mathbf{R}_{L P}^{*}$ are equal. $\mathbf{R}_{P}^{*}$ corresponds to a PIP flexed at about $90^{\circ}$

the degree that $c_{I L}>0$. In the normal finger the extrinsic effect $\left(1-X_{L}\right)$ dominates the intrinsic effect $\left(X_{L}\right)$ since $c_{I P}^{*} \gg c_{I L}$.

To summarize, lumbrical action can be seen as consisting of two superimposed effects: (i) the decrease of the extrinsic collapse force by decreasing $P_{\mathrm{d}}$, and (ii) the balancing of the remaining extrinsic collapse tendency by a true interosseus action. This view is illustrated in Fig. B1. where these two effects are represented by two separate motors which act with the same force. One motor deviates deep flexor force to the environment proximal to the MCP (in the Fig. B1(b) this effect is represented by introducing an exact antagonist $\mathbf{R}_{P L}=-\mathbf{R}_{P}^{*}$ of the deep flexor). The other motor is an interosseus with lumbrical moment arms. The Fig. B1(a) is kinematically consistent with the Fig. 1(a) or Fig. A1(a) when the displacements of both motors are mathematically added. 